VGDF, symptoms were reported more often by those with adult diagnosis. The results indicate the importance of targeted asthma treatment and follow-up by patient's exposure history and asthma diagnosis age.

\section{P54 COMBINED EXPOSURE TO VAPORS, GASES, DUSTS, FUMES AND TOBACCO SMOKE INCREASES THE RISK OF ASTHMA SYMPTOMS}

${ }^{1} \mathrm{H}$ Hisinger-Molkanen, ${ }^{2} \mathrm{P}$ Pallasaho, ${ }^{1,3} \mathrm{~A}$ Sovijarvi, ${ }^{4} \mathrm{~L}$ Tuomisto, ${ }^{5} \mathrm{H}$ Andersen, ${ }^{6} \mathrm{~A}$ Lindqvist, ${ }^{7} \mathrm{H}$ Backman, ${ }^{8,9} \mathrm{~A}$ Langhammer, ${ }^{7} \mathrm{E}$ Ronmark, ${ }^{10} \mathrm{~B}$ Lundback, ${ }^{4,11} \mathrm{P}$ Ilmarinen, 4,10,11 $\mathrm{H}$ Kankaanranta, ${ }^{1,3 \mathrm{P}}$ Piirila. 'University of Helsinki, Helsinki, Finland; ${ }^{2}$ Espoo City Health Services, Espoo, Finland; ${ }^{3}$ Unit of Clinical Physiology, HUS Medical Imaging Center, Helsinki University Central Hospital, Helsinki, Finland; ${ }^{4}$ Department of Respiratory Medicine, Seinäjoki Central Hospital, Seinäjoki, Finland; ${ }^{5}$ Karolinska University Hospital, Thoracic Oncology Unit, Tema Cancer, Stockholm, Sweden; ${ }^{6}$ Clinical Research Unit of Pulmonary Diseases, Helsinki University Hospital, Helsinki, Finland; ' Department of Public Health and Clinical Medicine, Division of Occupational and Environmental Medicine/the OLIN Unit, Umeå University, Umeå, Sweden; ${ }^{8}$ HUNT Research Centre, Department of Public Health and Nursing, NTNU, Norwegian University of Science and Technology, Levanger, Norway; ${ }^{9}$ Levanger Hospital, Nord-Trondelag Hospital Trust, Levanger, Norway; ${ }^{10}$ Krefting Research Centre, Institute of Medicine, University of Gothenburg, Gothenburg, Sweden; " ${ }^{11}$ Faculty of Medicine and Health Technology, Tampere University, Tampere, Finland

\subsection{6/thorax-2021-BTSabstracts. 164}

Background Occupational exposure to vapors, gases, dusts and fumes (VGDF) is known to increase the prevalence of asthma symptoms. Less is known about the prevalence of asthma symptoms if VGDF exposure is combined to smoking or environmental tobacco smoke exposure.

Objective To test the hypothesis that combined exposure to VGDF and tobacco smoke would have an additive effect on the risk for being symptomatic in responders with physician diagnosed asthma.

Methods A random sample of 16000 adults aged 20 to 69 years were invited to a postal survey on obstructive pulmonary diseases in Finland in 2016. Those who reported physician diagnosed asthma were included in the analysis and their reported VGDF exposure and smoking habits were analyzed. Being symptomatic was defined as an affirmative answer to three or more questions of asthma symptoms.

Results 8199 (51.5\%) subjects responded. Of the responders, 831 reported physician-diagnosed asthma. 22.3\% of asthmatics reported current smoking, 23.2\% exposure to environmental tobacco smoke and $39.4 \%$ occupational exposure to VGDF. $14.0 \%$ reported combined exposure to environmental tobacco smoke and VGDF, and 10.2\% exposure to VGDF and smoking. The prevalence of being symptomatic was increased in smokers $(73.0 \%$ vs $58.0 \%, \mathrm{p}=0.005)$ and in responders with occupational exposure to VGDF $(75.2 \%$ vs $58.0 \%, \mathrm{p}<0.001)$ compared to unexposed asthmatics. The highest prevalence estimates were seen in smokers with VGDF exposure $(83.5 \%$ vs $58.0 \%, \mathrm{p}<0.001)$ and in responders with exposure to both VGDF and environmental tobacco smoke $(85.3 \%$ vs $58.0 \%$, $\mathrm{p}<0.001$ ) suggesting their additive effect on the prevalence of asthma symptoms. There was no difference in asthma medication use between responders with no exposure history, smokers or those with environmental tobacco smoke or occupational VGDF exposure.

Conclusion Our results indicate an increased prevalence of asthma symptoms in adult asthmatics with exposure to VGDF and tobacco smoke. Asthmatics with exposure to both environmental tobacco smoke and VGDF had the highest prevalence estimates of asthma symptoms suggesting an additive effect. These results suggest the importance of prevention of occupational airborne exposures and smoking cessation in asthma treatment.

\section{P55 OCCUPATIONS, WORKPLACE EXPOSURES AND PHYSICAL DEMANDS OF WORK IN PATIENTS WITH SEVERE ASTHMA}

${ }^{1} \mathrm{GI}$ Walters, ${ }^{2} \mathrm{~F}$ Rezai, ${ }^{3} \mathrm{~N}$ Le Moual, ${ }^{1} \mathrm{~J}$ Marsh, ${ }^{1} \mathrm{~A}$ Bahron, ${ }^{2} \mathrm{MT}$ Krishna, ${ }^{1} \mathrm{AH}$ Mansur. ${ }^{1}$ University Hospitals Birmingham NHS Foundation Trust, Birmingham, UK; ${ }^{2}$ University of Birmingham, Birmingham, UK; ${ }^{3}$ Inserm UMR 1018 Centre for Epidemiology and Population Health, Paris, France

\subsection{6/thorax-2021-BTSabstracts. 165}

Introduction Patients with severe asthma are a group distinct from those with mild or moderate disease, due to their complex healthcare needs. Employment is common in this group, and complete work disability unusual. Asthma-related loss of productivity may be higher compared with patients with nonsevere asthma, and has been attributed to both physical and psychological impairments. We aimed to gain an understanding of the nature of work undertaken by patients with severe asthma.

Methods We searched the Birmingham (UK) Regional Severe Asthma Service (BRSAS) Dendrite clinical registry and included baseline demographic, current employment, and asthma-specific health data on employed patients (see figure). Recorded occupations for each included patient were processed by: (i) assigning a 4-digit SOC-2010 code, (ii) applying the OASJEM (job exposure matrix ${ }^{1}$ ) to determine likelihood of exposure to airway irritants and respiratory sensitizers, and (iii) assigning a 'physical demand' category (sedentary to very-heavy), using the Dictionary of Occupational Titles $\left(\mathrm{DOT}^{2}\right)$.

Results Data from $\mathrm{n}=548$ patients were included $(53 \%$ of severe asthmatics were employed, $86 \%$ of those working fulltime); median age $=44 \quad(\mathrm{IQR}=33-52)$, females $=367 \quad(67 \%)$, Caucasian ethnicity $=453 \quad(84), \quad$ current smokers $=27 \quad(5)$, median IMD decile $=5 \quad(2-7)$, median $\mathrm{BMI}=30 \quad(25-35), \geq 1$

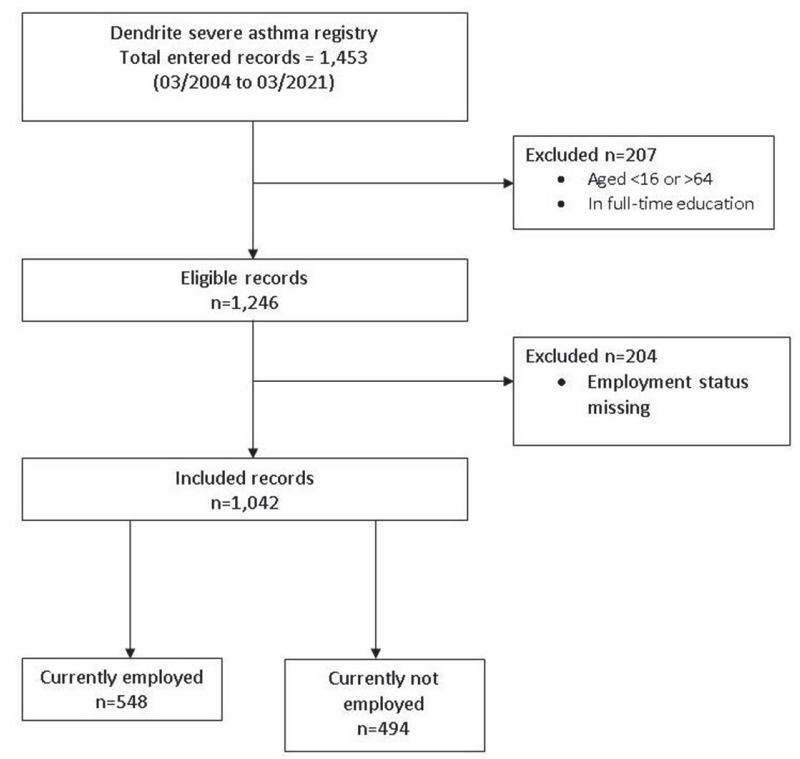

Abstract P55 Figure 1 